\title{
Analytical phase diagram for colloid-polymer mixtures
}

\author{
Gerard J. Fleer ${ }^{1}$ and Remco Tuinier ${ }^{2}$ \\ ${ }^{1}$ Laboratory of Physical Chemistry and Colloid Science, Wageningen University, 6703 HB Wageningen, The Netherlands \\ ${ }^{2}$ Forschungszentrum Jülich, Institut für Festkörperforschung, 52425 Jülich, Germany
}

(Received 23 April 2007; published 9 October 2007)

\begin{abstract}
We present a theoretical analysis of the phase behavior of colloid-polymer mixtures which applies to all polymer/colloid size ratios $q$. It accounts for the crossover from a constant length scale $R$ (radius of gyration) in the colloid limit (small $q$ ) to the concentration-dependent correlation length $\xi$ in the protein limit $(q>1)$. We obtain predictions that fully agree with observations and simulations. In the protein limit the colloid concentrations $\eta$ along the binodals become independent of $q$ and the polymer concentrations $\varphi$ scale as $q^{1 / \gamma}$, where $\gamma=0.77$ is the scaling exponent in $\xi \sim \varphi^{-\gamma}$ : phase diagrams plotted as $\varphi q^{-1 / \gamma}$ vs $\eta$ are then independent of $q$. The liquid window in the protein limit is narrow.
\end{abstract}

DOI: 10.1103/PhysRevE.76.041802

PACS number(s): 61.25.Hq, 82.70.Dd

\section{INTRODUCTION}

In biological and industrial dispersions such as food products [1] or the living cell [2] small colloids (proteins) and (bio)polymers are often jointly present. The stability of such mixtures depends on their concentrations and interactions. For nonadsorbing polymer, the colloid particles are surrounded by depletion layers [3-5]. This depletion arises from the configurational entropy loss of chains near a surface [6]. Overlap of depletion layers results in attractive forces $[3,4]$ between particles in colloidal [7] and biological systems such as actin networks [8,9] or virus dispersions [10]. Depletion forces induce phase transitions in colloid-polymer mixtures [11-13] which depend strongly on the size ratio $q$ $=R / a$, where $R$ is the radius of gyration of a polymer coil and $a$ the radius of a colloid particle.

In the so-called colloid limit (small $q$ ), the binodal polymer concentrations $\varphi$ are below overlap and the polymer length scale $R$ does not depend on $\varphi$. For this limit adequate theory is available [14-19] and the phase behavior is well understood. It resembles that of atomic fluids [20]: fluidsolid (FS) and gas-liquid (GL) phase transitions as well as critical points (cp) and triple points (tp) appear [11,12].

In the so-called protein limit $(q>1)$ the binodal polymer concentrations are in the semidilute regime. Here the length scale is the correlation length (blob size) $\xi$, which only depends on $\varphi$. For this limit the theoretical understanding [21-23] is much less satisfactory and phase diagrams can only be obtained numerically [24-26]. Scaling theory [27] predicts a wide liquid window for small spheres in a sea of semidilute chains. The underlying assumption that the blobs are much bigger than the colloids is, however, not supported by simulations [26] or by the theory proposed in this paper.

The protein limit is of great interest for nanoparticle engineering. Adding nonadsorbing polymers to protein solutions promotes protein crystallization [28-31]. Good-quality crystals are essential for studying protein structures by x-ray diffraction. Computer simulations [32] suggest that optimum protein crystallization conditions are correlated with nucleation from a condensed liquid of protein particles. This is only possible when GL coexistence is metastable: the stable liquid window should be narrow so that adding more poly- mer to proteins can push the system into the metastable GL regime where the crystalline state is stable.

In this paper we present a unified theory for colloidpolymer mixtures which includes the crossover in polymer length scales, from coil size to blob size. The GL region is much smaller than in existing theory because the blob size along the protein-limit binodals is smaller than the particle radius. We derive a scaling law that condenses these binodals for various size ratios onto a single curve.

\section{THEORY}

\section{A. Scaling}

The relevant parameters $[15,16,18,25]$ for colloid/polymer phase diagrams are the reduced polymer concentration $y$ $=\varphi / \varphi^{*}$ in an external reservoir, the colloid volume fraction $\eta$, and the size ratio $q=R / a$. Here $\varphi$ is the (external) polymer volume fraction and $\varphi^{*}$ the volume fraction at overlap. The radius of gyration $R$ scales as $N^{\nu}$, where $N$ is the chain length and $\nu \approx 0.59$ is the Flory exponent. We also introduce the size ratio $q_{s}=\delta_{s} / a$, where $\delta_{s}$ is the depletion thickness around a colloidal sphere. In our approach $q_{s}$ not only depends on $q$ but also on the polymer concentration: $q_{s}$ $=q_{s}(q, y)$, which captures the crossover in length scales, from the colloid limit (where $q_{s}$ is independent of $y$ ) to the protein limit (where $q_{s}$ becomes independent of $q$ ).

In the colloid limit the polymer concentrations at GL coexistence are below overlap $(y<1)$. In this dilute range the length scale $\delta_{s}$ is of order $R$ and independent of $y$. Then $q_{s}$ $\approx q$, which is the situation described by the free-volume theory (FVT) of Lekkerkerker et al. [15]. We denote this theory as FVTL.

In the protein limit $y$ along the binodals exceeds unity and we have semidilute polymer solutions, with length scale $\xi$ $\sim \varphi^{-\gamma}$ [5]. The de Gennes exponent $\gamma(=0.77)$ is directly coupled to the Flory exponent $\nu$ : $(3 \nu-1)(3 \gamma-1)=1$. The dilute length scale $R$ is in the same way related to $\varphi^{*}: \varphi^{*}$ $\sim N / R^{3} \sim N^{1-3 \nu} \sim R^{-1 / \gamma}$ or $R \sim\left(\varphi^{*}\right)^{-\gamma}$. The ratio $\xi / R$ is then only a function of $y: \xi / R \sim y^{-\gamma}$. It is important to note that $\xi$ is independent of $R$, which immediately leads to $y \sim R^{1 / \gamma}$.

The scaling relation for $\xi / R$ in semidilute solutions may be directly translated towards the ratio $q_{s} / q$ in the protein 


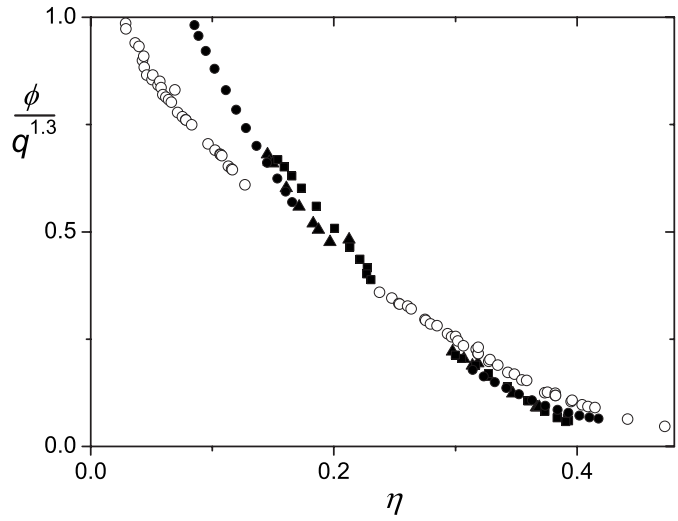

FIG. 1. Monte Carlo simulation data by Bolhuis et al. $[19,26]$ replotted in the form $\phi q^{-1.3}$ versus the colloid volume fraction $\eta$, for $q=1.05$ (open circles), 3.86 (solid circles), 5.58 (solid triangles), and 7.78 (solid squares).

limit. In this limit $q_{s} \approx \xi / a$, so $q_{s} / q \approx \xi / R \sim y^{-\gamma}$. Just as $\xi$ is independent of $R, q_{s}=\xi / a$ is independent of $q=R / a$ :

$$
y \sim q^{1 / \gamma}
$$

This equation is a key element in our analysis; it applies generally to colloids in semidilute polymer solutions. We are particularly interested in $y^{c p}$ (critical point, $\mathrm{cp}$ ) and $y^{t p}$ (triple point, tp) since these determine the range over which a colloidal liquid is stable. The GL binodal $y(\eta)$ has its minimum $y^{c p}\left(\eta^{c p}\right)$ in the cp and its ends at $y^{t p}\left(\eta^{t G}\right)$ (gas side tG of tp) and at $y^{t p}\left(\eta^{t L}\right)$ (liquid side tL of tp). Clearly, the triple point has also a solid part, but we concentrate on GL coexistence. The important implication of Eq. (1) is that phase diagrams $y(\eta)$ become universal when plotted in terms of $y q^{-1 / \gamma}$ vs $\eta$.

Not only is $q_{s}$ (hence, $q_{s}^{c p}$ and $q_{s}^{t p}$ ) independent of $q$ in the protein limit but this applies also to the colloid concentrations $\eta^{t G}, \eta^{c p}$, and $\eta^{t L}$. The tG phase is very dilute in colloids $\left(\eta^{t G} \approx 0\right)$, and the tL phase is nearly void of polymer so that $\eta^{t L}$ is close to 0.49 , which is the fluid concentration for hardsphere (HS) fluid/solid coexistence in the absence of polymer [33]. Simulations [26] and integral theory [25] show that also $\eta^{c p}$ (somewhere between $\eta^{t G}$ and $\eta^{t L}$ ) becomes constant in the protein limit.

Equation (1) not only applies to the external (reduced) concentrations $y$ but also to the internal (reduced) concentrations $\phi=\alpha y$. Here $\alpha$ is the fraction of free volume (i.e., the fraction not occupied by the colloids plus depletion layers). It depends only on $q_{s}$ and $\eta$, which are independent of $q$ in the protein limit. Thus, also $\alpha^{t G}, \alpha^{c p}, \alpha^{t L}$, and the $\alpha$ 's along the binodal are then independent of $q$ : binodals $\phi(\eta)$ scale with $q$ just like binodals $y(\eta)$.

As an illustration of Eq. (1) we replot recent data of Bolhuis et al. $[19,26]$ in Fig. 1. These authors performed largescale Monte Carlo (MC) simulations for $q=1.05$ [19], 3.86, 5.58 , and 7.78 [26] and found that the various GL binodals $\phi(\eta)$ differ widely. When their results are replotted as $\phi q^{-1 / \gamma}$, as in Fig. 1, we see a nearly universal behavior. The binodals for the three highest $q$ values collapse onto a single curve; here we are truly in the protein limit. The binodal for $q=1.05$ (open circles) is a borderline case: it coincides approximately with the universal curve at the liquid side and deviates downwards at the gas side. For lower $q$ (not shown) stronger deviations are found since the protein limit is not yet reached and $q_{s}$ does still depend on $q$.

\section{B. Free-volume theory}

The challenge is to capture these findings in quantitative theory. For the colloid limit FVTL [15] is a fair approximation. The grand potential $\omega$ of a colloid/polymer mixture in equilibrium with an external reservoir containing only the polymer solution is separated in an HS part $\omega_{0}$ and a polymer contribution $\omega_{p}$. In the original FVTL the solvent is treated as background and $\omega_{p}$ is formulated in terms of the polymer concentration $\varphi$, the polymer chemical potential $\mu_{p}$, and the free-volume fraction $\alpha$ which depends on $q$ and on $\eta$ but not on polymer concentration; for the expression $\alpha(q, \eta)$, we refer to the literature [15]. We have shown recently [34] that the solvent (with reservoir volume fraction $1-\varphi$ ) can be taken explicitly into account. Through the Gibbs-Duhem rule $\mu_{p}$ may be related to the product $\Pi v$, which is the osmotic work to insert a colloid particle (without depletion layer) into the polymer solution; here, $\Pi$ is the external osmotic pressure and $v$ the volume of a colloid particle. The expression for $\omega_{p}$ now takes a simple and elegant form

$$
\omega_{p}=-\int_{0}^{y} \alpha(\partial \Pi v / \partial y) d y .
$$

In FVTL $\alpha$ does not depend on $\Pi$ or $y$, so Eq. (2) simplifies to $\omega_{p}=-\alpha \Pi v$. For the sake of later generalization we keep the integral form of Eq. (2).

In dilute polymer solutions $\Pi=\varphi / N$ and $\Pi v$ may be simply expressed in $q$ and $y$. With $\varphi^{*}=(3 / 4 \pi) N / R^{3}$ and $v$ $=(4 \pi / 3) a^{3}$ we obtain $\Pi v=q^{-3} y$, which leads to Eq. (3a). In the original FVTL curvature effects are ignored: the depletion thickness $\delta_{s}$ around a sphere is set equal to $\delta_{p} \approx R$ next to a flat plate. Hanke et al. [35] established the relation between $\delta_{s}$ and $\delta_{p}$ or, equivalently, between $q_{s}$ and $q$. To a very good approximation this relation may be written as Eq. (3b):

$$
\begin{gathered}
\partial \Pi v / \partial y=q^{-3}, \\
q_{s}=0.865 q^{0.88} .
\end{gathered}
$$

So now $\alpha$ in Eq. (2) is $\alpha\left(q_{s}, \eta\right)$. Henceforth we use the acronym FVTL for the version where curvature effects are accounted for by Eq. (3b): $q_{s}$ differs from $q$ but is still of the same order of magnitude and independent of $y$. Inserting Eqs. (3) into Eq. (2), in combination with the standard expressions for the free energy of an HS fluid or HS solid, gives the grand potential $\omega(q, y, \eta)$ for a colloid-polymer mixture, from which the complete phase diagram may be calculated. Binodals, triple, and critical GL points follow from standard thermodynamics and are obtained analytically. Also the critical end point (cep) [34], which marks the lowest $q=q^{c e p}(\approx 0.35)$ where a colloidal liquid is stable, is found directly. At the cep the critical (GL) point coincides with the triple (GLS) point. When $q$ exceeds $q^{c e p}$, both $\mathrm{cp}$ and tp 


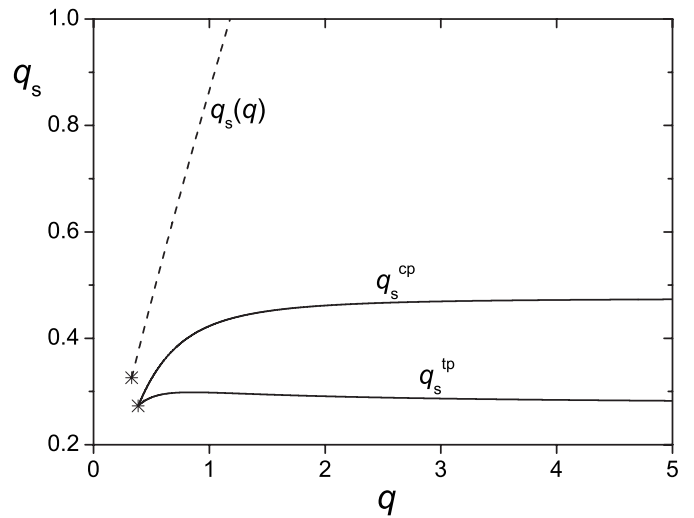

FIG. 2. The dependence of $q_{s}^{c p}$ (critical point) and $q_{s}^{t p}$ (triple point) on the size ratio $q$ (solid curves). The dashed curve is $q_{s}(q)$ in FVTL. The asterisks are the critical end points in the two models.

change: this defines the critical curve $\operatorname{cp}(q)$ and the triple curves $\operatorname{tp}(q)$. The analysis provides both the critical and triple curves analytically.

\section{Generalized free-volume theory}

FVTL breaks down when $q$ exceeds 0.5 because then $y$ along the binodals exceeds unity, and Eq. (3a) for $\Pi v$ is inadequate. Moreover, $q_{s}$ is no longer of order $q$ and depends on $y$. We can still use Eq. (2) with $\alpha\left(q_{s}, \eta\right)$ but we need new relations for $q_{s}(q, y)$ and $\Pi v(q, y)$. We start from the semidilute limit where the length scale is $\xi$, substituting $\xi / a$ for $q$ in Eq. (3b). We have shown before [36] that $\xi / \delta_{0}=0.50 y^{-\gamma}$; the prefactor comes from simulations [37] and $\delta_{0}=1.071 R$ [35] is the dilute limit of $\delta_{p}$. Now $q$ in Eq. (3b) is replaced by $0.50 q y^{-\gamma}$. In combination with Eq. (1) this gives a value of $q_{s}$ which is independent of $q$, as expected. For $\Pi v$ we use a renormalization-group result [38] $\Pi / \Pi_{0}=1.62 y^{3 \gamma-1}$, where $\Pi_{0} v=q^{-3} y$ is the dilute limit of Eq. (3a). This leads to $\partial \Pi v / \partial y=3.77 q^{-3} y^{3 \gamma-1} ; \Pi v \sim q^{-3} y^{3 \gamma}$ is again independent of $q$.

The next step is the crossover between the dilute and semidilute limits. We use a linear combination $\Pi=\Pi_{0}+\Pi_{s d}$ of the two limits for the osmotic pressure $\Pi$ and an inversesquare combination $\delta_{p}^{-2}=\delta_{0}^{-2}+\xi^{-2}$ for the depletion thickness $\delta_{p}$ [39]. These combination rules are in excellent agreement with simulations and with experiment [36]. Now Eqs. (3) are generalized to

$$
\begin{gathered}
\partial \Pi v / \partial y=q^{-3}\left[1+3.77 y^{3 \gamma-1}\right], \\
q_{s}=0.865\left(q / \sqrt{1+3.95 y^{2 \gamma}}\right)^{0.88} .
\end{gathered}
$$

For small $y$, Eqs. (3) are recovered and for high $y$ we reach the semidilute limits discussed above. Inserting Eqs. (4) and (5) into Eq. (2) enables the calculation of phase diagrams. We note that $q_{s}$ is small $(<1)$ even in the protein limit so that the expression for $\alpha\left(q_{s}, \eta\right)$, which agrees with simulations for $q_{s}$ up to $O(1)$, remains valid, unlike in FVTL where $q_{s}$ $\approx q$ diverges with $q$.

Figure 2 (solid curves) gives the dependence of $q_{s}^{c p}$ and $q_{s}^{t p}$ on $q$. The point to the left where the two curves meet

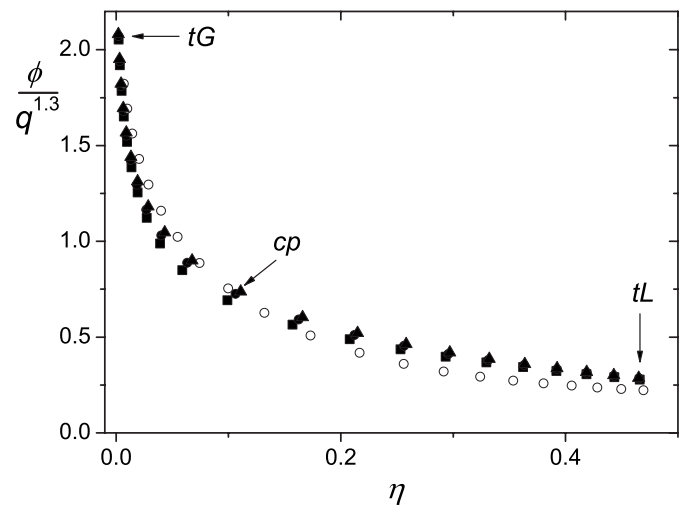

FIG. 3. Analytical binodals in the form $\phi q^{-1.3}$ versus the colloid volume fraction $\eta$, for $q=1$ (open circles), 3 (solid circles), 4 (solid triangles), and 5 (solid squares). The triple and critical points are indicated.

(indicated by the asterisk) is the cep; its coordinates are

$$
\begin{aligned}
& q^{c e p}=0.39, q_{s}^{c e p}=0.27, \quad y^{c e p}=0.43, \\
& \alpha^{c e p}=0.38, \quad \phi^{c e p}=0.16, \quad \eta^{c e p}=0.32 .
\end{aligned}
$$

For comparison we show in Fig. 2 also $q_{s}(q)$ for FVTL (dashed line) according to Eq. (3b). In this case there is no difference between $q_{s}^{c p}$ and $q_{s}^{t p}$ since the $y$ dependence in Eq. (5) is neglected. The cep in both models is about the same (in FVTL $q^{c e p} \approx q_{s}^{c e p}=0.33, y^{c e p}=0.39$ ) because it is (more or less) in the dilute regime. Recently, we discussed the implications of the cep in some detail and made a comparison with a one-component Yukawa fluid [34,40].

\section{Protein limit}

Next we focus on the protein limit. For high $q, q_{s}^{c p}$ and $q_{s}^{t p}$ in Fig. 2 reach a final plateau, as expected. The results for $\mathrm{cp}$ and tp in the protein limit are

$$
\begin{gathered}
q_{s}^{c p}=0.47, \quad y^{c p}=1.03 q^{1 / \gamma}, \quad \alpha^{c p}=0.71, \\
\phi^{c p}=0.73 q^{1 / \gamma}, \quad \eta^{c p}=0.11, \\
q_{s}^{t p}=0.28, \quad y^{t p}=2.18 q^{1 / \gamma}, \\
\alpha^{t G}=1, \quad \phi^{t G}=2.18 q^{1 / \gamma}, \quad \eta^{t G}=0.0022, \\
\alpha^{t L}=0.14, \quad \phi^{t L}=0.30 q^{1 / \gamma}, \quad \eta^{t L}=0.47 .
\end{gathered}
$$

Except for the internal and external polymer concentrations, which scale as $q^{1 / \gamma}$, all other characteristics of the critical and triple points become $q$ independent in the protein limit. Hence, when the polymer concentrations are rescaled by multiplying them by $q^{-1 / \gamma}$, universal properties are obtained. For simulations [26] this was already demonstrated in Fig. 1. Figure 3 gives our analytical predictions, for $q=1$ (borderline case) and $q=3,4,5$ (protein limit). For the region $\phi q^{-1.3}$ below 1 (the range in Fig. 1), the overall picture in Figs. 1 and 3 is the same. In Fig. 3 the $L$ branch is somewhat flatter than in the simulations. 
So we obtain explicit expressions for the three characteristic points of a GL binodal (tG, cp, and tL) [Eqs. (7)-(10)]. When we take cp as the reference point, in the protein limit the external polymer concentrations for a stable colloidal liquid are in the range $y^{c p}<y<2 y^{c p}$, and the colloid concentrations in the range $0<\eta<0.11$ (gas branch) or $0.11<\eta$ $<0.47$ (liquid branch). In terms of the internal polymer concentrations the gas branch is in the range $3 \phi^{c p}>\phi>\phi^{c p}$, and for the liquid branch $\phi^{c p}>\phi>0.4 \phi^{c p}$. The liquid window is thus narrow.

We may compare the results for $\mathrm{cp}$ in Eqs. (7) with simulations [26] and recent perturbation theory [41]. The colloid concentration $\eta^{c p}=0.11$ agrees nearly quantitatively with perturbation theory, simulations giving a higher value $(\approx 0.25)$. The scaling $\phi^{c p} \sim q^{1 / \gamma}$ is accurately reproduced in both simulations and perturbation theory. There is a slight discrepancy as to the prefactor.

In practice colloids are polydisperse and this suppresses freezing of a colloidal dispersion, which widens the colloidal liquid window. Globular proteins are monodisperse and adding long polymer chains to their solutions might therefore indeed facilitate protein crystallization: it is easy to add so much polymer that one enters the metastable regime of GL coexistence where protein crystals are stable [32].

\section{CONCLUSIONS}

Analytical theory was presented for the phase behavior of a dispersion of hard-sphere colloids plus nonadsorbing polymer with excluded-volume interactions, for arbitrary size ratio $q=R / a$. We find nearly quantitative agreement with computer simulations in the protein limit. In this limit the blob size along the binodals is smaller than the particle radius. When the polymer concentrations in critical and triple points are normalized by multiplying them by $q^{-1.3}$ universal properties are found. The liquid window is narrow, which helps in understanding why adding polymer to small colloids like proteins promotes crystallization.

\section{ACKNOWLEDGMENTS}

We thank N. A. M. Besseling and F. A. M. Leermakers for a critical reading of the manuscript and P. G. Bolhuis for sending us his MC data.
[1] J.-L. Doublier et al., Curr. Opin. Colloid Interface Sci. 5, 184 (2000).

[2] S. B. Zimmerman and A. P. Minton, Annu. Rev. Biophys. Biomol. Struct. 22, 27 (1993).

[3] S. Asakura and F. Oosawa, J. Chem. Phys. 22, 1255 (1954).

[4] A. Vrij, Pure Appl. Chem. 48, 471 (1976).

[5] P. G. De Gennes, Scaling Concepts in Polymer Physics (Cornell University Press, Ithaca, NY, 1979).

[6] E. Eisenriegler, J. Chem. Phys. 79, 1052 (1983).

[7] R. Verma, J. C. Crocker, T. C. Lubensky and A. G. Yodh, Phys. Rev. Lett. 81, 4004 (1998).

[8] M. Hosek and J. X. Tang, Phys. Rev. E 69, 051907 (2004).

[9] R. Tharmann et al., Biophys. J. 90, 2622 (2006).

[10] Z. Dogic, K. R. Purdy, E. Grelet, M. Adams, and S. Fraden, Phys. Rev. E 69, 051702 (2004).

[11] S. M. Ilett, A. Orrock, W. C. K. Poon, and P. N. Pusey, Phys. Rev. E 51, 1344 (1995).

[12] A. Moussaïd, W. C. K. Poon, P. N. Pusey, and M. F. Soliva, Phys. Rev. Lett. 82, 225 (1999).

[13] W. C. K. Poon, J. Phys.: Condens. Matter 14, R859 (2002).

[14] A. P. Gast et al., J. Colloid Interface Sci. 96, 251 (1983).

[15] H. N. W. Lekkerkerker et al., Europhys. Lett. 20, 559 (1992).

[16] E. J. Meijer and D. Frenkel, J. Chem. Phys. 100, 6873 (1994).

[17] M. Dijkstra, R. van Roij, R. Roth, and A. Fortini, Phys. Rev. E 73, 041404 (2006).

[18] D. G. A. L. Aarts et al., J. Phys.: Condens. Matter 14, 7551 (2002).

[19] P. G. Bolhuis, A. A. Louis, and J. P. Hansen, Phys. Rev. Lett. 89, 128302 (2002).

[20] W. C. K. Poon et al., Phys. World 9 (4), 27 (1996).
[21] K. Mutch et al., Soft Matter 3, 155 (2007).

[22] T. Odijk, J. Chem. Phys. 106, 3402 (1997).

[23] R. P. Sear, Phys. Rev. Lett. 86, 4696 (2001).

[24] M. Fuchs and K. S. Schweizer, Europhys. Lett. 51, 621 (2000).

[25] M. Fuchs and K. S. Schweizer, J. Phys.: Condens. Matter 14, R239 (2002).

[26] P. G. Bolhuis, E. J. Meijer, and A. A. Louis, Phys. Rev. Lett. 90, 068304 (2003).

[27] P. G. de Gennes, C. R. Seances Acad. Sci., Ser. B 288, 203 (1979).

[28] S. Finet and A. Tardieu, J. Cryst. Growth 232, 40 (2001).

[29] A. Polson et al., Biochim. Biophys. Acta 82, 463 (1964).

[30] A. M. Kulkarni, A. P. Chatterjee, K. S. Schweizer, and C. F. Zukoski, Phys. Rev. Lett. 83, 4554 (1999).

[31] S. Ataka and M. Ataka, J. Chem. Phys. 117, 3504 (2002).

[32] P. R. Ten Wolde and D. Frenkel, Science 277, 1975 (1995).

[33] W. G. Hoover and F. M. Ree, J. Chem. Phys. 49, 3609 (1968).

[34] G. J. Fleer and R. Tuinier, Physica A 379, 52 (2007).

[35] A. Hanke, E. Eisenriegler, and S. Dietrich, Phys. Rev. E 59, 6853 (1999).

[36] G. J. Fleer et al., Macromol. Theory Simul. 16, 531 (2007).

[37] A. A. Louis et al., J. Chem. Phys. 116, 10547 (2002).

[38] L. Schäfer, Excluded Volume Effects in Polymer Solutions (Springer-Verlag, Heidelberg, 1999).

[39] G. J. Fleer et al., Macromolecules 36, 7857 (2003).

[40] R. Tuinier and G. J. Fleer, J. Phys. Chem. B 110, 20540 (2006).

[41] A. Pelissetto and J.-P. Hansen, Macromolecules 39, 9571 (2006). 